\title{
Die Therapie der Pariser Kliniker zwischen 1795 und 1840
}

\author{
Von Erwin H.AcKerknecht
}

Während man im deutschen Sprachgebiet mit dem Werk von PAsteur, Charcot oder Claude Bernard wohl vertraut scheint, ist die Geschichte der Pariser Klinik in der ersten Hälfte des 19. Jahrhunderts relativ wenig bekannt. Das liegt wohl vor allem daran, daß es im Gegensatz zur BernardPasteur-Periode in der Geschichte der deutschen Medizin keine der Pariser Klinik entsprechende schöpferische Periode gibt ${ }^{1}$. In dieser Zeit war östlich des Rheins ja vor allem das Knaben-Wunderhorn der Romantik zu vernehmen. Da es sich bei der Pariser Schule des Jahrhundertbeginns um eines der wichtigsten Phänomene der Medizingeschichte handelt ${ }^{2}$, scheint ein genaueres Studium aller Aspekte derselben, auch ihrer Therapie, sehr wünschenswert. Die Therapie ist zwar (wie meist) nicht der Glanzpunkt der Geschichte der Schule, der bei der Diagnostik und Pathologie liegt, aber auch sie sollte einmal untersucht werden. Denn eine gesteigerte Beschäftigung mit der Geschichte der Theapie, die, obwohl praktisch vielleicht der wichtigste Teil der Medizingeschichte, im allgemeinen recht stiefmütterlich behandelt wird, scheint nicht minder notwendig.

Es ist bekannt, daß Frankreich um 1790 zusammen mit seiner politischen auch eine medizinische Revolution erlebte. Der schon von MoLIÈre verspottete traditionelle Humoralismus und Systematismus wurde radikal durch einen lokalistischen Solidismus ersetzt, der sich auf eine neue Art der Beobachtung stützte. Diese ist vor allem durch die neuen physikalischen Untersuchungsmethoden, die Leichenöffnung und Statistik charakterisiert. Der praktischen Neuorientierung ging eine philosophische voraus, formuliert vor allem durch Cabanis. Seine sensualistische Philosophie der Beobachtung war verbunden mit einem tiefen Mißtrauen gegen alles Nichtbeobachtete, d.h. Theorien, und gegen die Übertragung nichtklinischer Beobachtungen aus dem Gebiet der Chemie, der Experimentalphysiologie

1 Selbst Sснӧnlein, der Schüler DöLlingers, über den wir vor allem verweisen auf W.Löfflers Studie in Zürcher Spitalgeschichte, Zürich 1951, Band 2, S. 2-90, kann nur bedingt in diesen Kreis gerechnet werden.

${ }^{2}$ Eine kurze Zusammenfassung meiner langjährigen Forschungen auf diesem Gebiet findet sich in: E.H.Ackerknecht, La Médecine à Paris entre 1800 et 1850, Conférences du Palais de la Découverte, Série D No 58, Paris 1958, 21 p. 
und der Mikroskopie auf die Klinik. Die Pariser Medizin entwickelte sich also weder in Laboratorien, wie unsere eigene, noch am individuellen Krankenbett oder in der Bibliothek wie ihre Vorgänger, sondern in den großen Spitälern der Seinestadt. Sie kann darum als besonderer Typus, als «Spitalmedizin», angesprochen werden. Es ist kein Zufall, daß die Blüte der Spitalmedizin in Paris auch mit einer Blüte der Spitalpharmazie zusammenfällt, in der Frankreich allen Ländern weit vorauseilte ${ }^{3}$.

Die radikale Umwertung aller Werte durch die Pariser Ärzte um 1800 brachte auf therapeutischem Gebiet vor allen Dingen den Skeptizismus hervor (mit Ausnahme der Schule von Broussars), führte einige von ihnen aber noch einen Schritt weiter, nämlich zur Schaffung der modernen Pharmakologie, zu einer auf das Tierexperiment gestützten Drogentherapie. Der therapeutische Skeptizismus ist, wie wir im Detail noch sehen werden, keineswegs gleichbedeutend mit dem therapeutischen Nihilismus, wie er sich dann unter SкорA und Diets in Wien in den vierziger Jahren des neunzehnten Jahrhunderts entwickelte ${ }^{4}$. Skeptizismus bedeutet allerdings bereits verminderte Verwendung oder Abschaffung zahlreicher als unwirksam erkannter Medikamente ${ }^{5}$ und Methoden, und entsprechend größeres Vertrauen in die Heilkraft der Natur. Er bedeutet Betonung der «empirischen» und Ablehnung der «rationalen» Therapie. Eine «rationale» d.h. eine auf einer physiologisch-pathologischen Theorie aufgebaute Therapie war nach Cabanis und seinen Nachfolgern ausgeschlossen, da nach ihm

${ }^{3}$ Zur Geschichte der Spitalspharmazie siehe: A.Berman, Tradition and Change: The Hospital Formulay in the US, J. Mond. de Pharmacie, Sept. 1957. Derselbe, Education for Hosp. Pharmacy, Bull. Am. Soc. of Hosp. Pharm. 12 (1955) 248-53. Derselbe, Ratier's Formulary of the Parisian Hospitals, a.a.O. 13 (1956) 462-64. Zur Geschichte der Pariser Spitalspharmazie: A.Gonis, Centenaire de l'Internat en Pharmacie des Hôpitaux de Paris, Paris 1920. M. Bouvet, La Pharmacie Hospitalière à Paris de 1789 à 1815, Paris 1943. R. Hazard, L'Internat en Pharmacie dans les Hôpitaux de France, La Pharmacie Française 55 (1951) 115.

4 J.Petersen, Hauptmomente in der geschichtlichen Entwicklung der medizinischen Therapie, Kopenhagen 1877, S. 184-210. Skoda selbst war kein Nihilist, s. E. LESKY, Wiener Med. Wschr. 68 (1956) 728.

${ }^{5}$ Die radikalsten Formulierungen auf diesem Gebiet stammen von den amerikanischen Sprößlingen der Pariser Schule, wie Elisha Bartlett, der 19/20 aller Medikamente abschaffen wollte (An Essay on the Philosophy of Medical Science, Philadelphia 1844, S. 286) oder Oliver Wendell Holmes, der, nachdem er ein halbes Dutzend Medikamente ausgenommen hatte, feststellte: "I firmly believe that if the whole materia medica, as now used, could be sunk to the bottom of the sea, it would be all the better for mankind-and all the worse for the fishes" (Medical Essay, Boston, S. 203). 
echte Kausalkenntnis über die Wirkung der Medikamente nicht möglich und auch nicht nötig war. Die Therapie konnte nur aus der Therapie abgeleitet werden d.h. konnte also nur empirisch $\operatorname{sein}^{6}$.

Der Skeptizismus richtete sich vor allem gegen die Drogentherapie, die Materia medica, die mehr und mehr die Therapie geworden war. Er brachte darum einen erweiterten Therapiebegriff hervor, der in gewissem Maß eine Rückkehr zu den hippokratisch-galenischen Traditionen darstellt und in dem die Drogentherapie wieder nur einer der verschiedenen Zweige der Therapie ist. Dies spiegelt sich in den Therapiedefinitionen der Periode wieder. Schwilgué, das therapeutische Sprachrohr Pinels, unterteilt die Therapie in "Hygiene» und Materia medica ${ }^{7}$. G. L.BAYLE, dasjenige Mitglied der Corvisart-Schule, das sich am ausführlichsten zur Therapie geäußert hat, gibt Materia medica, Pharmazie, Chirurgie und Hygiene als Unterabteilungen an ${ }^{8}$. J.B.G.Barbier D'Amiens, der Bichats Arbeiten auf dem Gebiet der Materia medica fortsetzt, weist in seinem Brief vom 21. März $1822 \mathrm{im}$ 60. Band des Dictionnaire des Sciences Medicales auf die Schwierigkeiten über Therapie zu schreiben hin, nachdem das Symptom von der Läsion als pathologischer Grundbegriff ersetzt worden ist - wie soll man eine Pharmakologie der Läsionen schreiben? - und unterscheidet Hygiene, Materia medica und physikalische Methoden in der Therapie. L.B.Guersant schließlich, im Artikel «Thérapeutique» im Band 29 des Dictionnaire de Médecine (1844) nennt physikalische und chemische Mittel (Wasser, Elektrizität usw.), Chirurgie, Hygiene, untergeteilt in Diät und «moralische Mittel», und Medikamente.

Der Begriff der Hygiene, der hier und andernorts im französischen Schrifttum der Zeit so oft auftaucht, ist vieldeutig. Er bezieht sich auf Vorbeugungsmaßnahmen aller Art $^{9}$ sowie auf nicht arzneiliche (Diät, Wärme, Wasser, Elektrizität usw.) oder psychische Therapie. Die positive Seite des Skeptizismus war, daß die überragende Bedeutung der Vorbeugung wieder erkannt, und lange vernachlässigte Methoden wie Freiluft-

${ }^{6}$ E.H.Ackerknecht, Elisha Bartlett and the Philosophy of the Paris Clinical Schoo 1 Bull. Hist. Med. 24 (1950) 52.

7 Traité de matière médicale, Paris 1805.

${ }^{8}$ Idée générale de la thérapeutique, Biblioth. méd. t. X, Paris 1805, S. 290. Nachgedruckt in Euvres de Corvisart, Auenbrugger, Bayle, Paris 1855.

9 Aus ihrem therapeutischen Skeptizismus heraus wurden die Franzosen führend auf dem Gebiet der Hygiene, siehe E.H.Ackerknecht, Hygiene in France 1815-1848. Bull. Hist. Med. 22 (1958) 117-55. 
behandlung, Gymnastik, Wasser-, Hitze-, Kälte- und psychische Behandlung wieder belebt wurden ${ }^{9 a}$.

Gerade auf therapeutischem Gebiet wurde die Pariser Schule viel im In- und Ausland angegriffen ${ }^{10}$. Man warf ihr vor, die Therapie im allgemeinen zu vernachlässigen, besonders aber die Allgemeinbehandlung und das Individualisieren in der Therapie. Es ist unleugbar, daß sich das Interesse der Schule mehr auf die Pathologie, besonders die pathologische Anatomie, als - mit Ausnahme der Chirurgie - auf die Therapie konzentrierte. Aber es war eben vor allem notwendig, erst einmal festen Boden unter den Füßen zu gewinnen, erst zu wissen, was man behandelte, eh man erforschen konnte, wie man behandeln solle. Besonders nachdem sich herausgestellt hatte, daß die meisten der traditionellen Behandlungsmethoden durchaus nicht hielten, was sie versprachen. Rostan stellte trotz dieser angeblichen Vernachlässigung der Therapie eine Halbierung der Sterblichkeit im Hôtel-Dieu zwischen 1789 und 1844 fest und argumentierte: «La médecine du symptome sans la connaissance du diagnostic local est une chose absurde, parfaitement impuissante et quelquefois nuisible et meurtrière ${ }^{11}$. Viel zutreffender sind die Vorwürfe betreffend Allgemeinbehandlung und Individualisieren. Der pathalogisch-anatomische Lokalismus der Pariser Schule mußte der Entwicklung der Allgemeinbehandlung ungünstig sein, ihre «Ontologie», ihr Bestreben feste Krankheitsbilder, wie Tuberkulose, Diphtherie usw. zu schaffen, das sie mehr an der Krankheit als am Kranken interessiert sein ließ, mußte dem Individualisieren ebenso abträglich, wie der Vorliebe für Spezifika zuträglich sein. Der Lokalismus brachte andererseits ein Aufblühen der Chirurgie hervor. So sehr der Skeptizismus und Lokalismus auf die Dauer in eine Sackgasse führen mußte, so muß er doch als eine wichtige, notwendige und positive Etappe in der Entwicklung der Therapie angesehen werden ${ }^{11 \text { a }}$.

${ }^{9 a}$ Für Details siehe H.Grasset, La médecine naturaliste à travers les siècles, Paris 1911; P.Delaunay, D'une révolution à l'autre. L'évolution des théories et pratiques médicales 1789-1849, Paris 1949.

10 Siehe z.B. J.B. Cayol, Clinique médicale, Paris 1833; J.L.Brachet, De l'emploi de l'opium dans les phlegmasies, Lyon 1828; Cless-Stuttgart, La médecine en France, Arch. gén. de la méd., t. 52 (1840) 377; E. Kratzmann, Die neuere Medizin in Frankreich, Leipzig 1846; C.A. Wunderlich, Geschichte der Medizin, Stuttgart 1859, p. 318; Bouchut bei Petersen, l.c., S. 181.

11 De l'organicisme, Paris 1864, S. 75, 375.

11a E. H.Acкerknecht, Wendepunkte in der Geschichte der Pharmakotherapie, Schweiz. Apotheker-Ztg. 95 (1957) 755. 
Nach diesen allgemeinen Feststellungen scheint es wünschenswert, sich etwas konkreter mit den therapeutischen Ideen wenigstens der hervorragendsten Vertreter der Schule zu beschäftigen. PINEL, von 1795 bis 1816 ihr unbestrittenes Haupt, zeigte ein «sentiment extrème de répugnance pour la polypharmacie ${ }^{12}$. In späteren Auflagen seiner Nosographie philosophique ${ }^{13}$ übernahm er wörtlich das vernichtende Urteil seines Schülers Brснат über die Materia medica seiner Zeit: «ensemble informe d'idées inexactes, d'observations souvent puériles, de moyens illusoires ${ }^{14}$. Was waren seine Reformvorschläge? «Simplifier au dernier degré la matière médicale, la réduire à l'usage d'un petit nombre de plantes indigènes d'une vertu constatée ou à des substances chimiques simples ou très-peu composées ${ }^{15}$. «Les règles vraies du traitement ne doivent-elles pas être immédiatement déduites de la marche et de la nature des symptomes, et modifiées suivant les variétés accessoires des maladies? ${ }^{16}$ Pinel war zweifellos Skeptiker, schwankte aber scheinbar zwischen «empirischer» und «rationaler» Therapie. Er löste das Problem auf die allereinfachste Weise, wie ein Blick auf seine Krankengeschichten zeigt: er gab gar nichts. Im Konflikt zwischen médecine d'action und médecine d'expeçtation optierte er resolut für die letztere, seine Hoffnung hauptsächlich auf die hippokratische Naturheilkraft setzend. «Ce serait un grand et beau sujet à traiter en médecine, que celui des maladies qui sont aggravées par un traitement inconsidéré et sans méthode, ou par un abus de remèdes, lorsqu'il aurait fallu se borner à une expectation sage et mesurée, ou, ce qui en diffère peu, à un usage extrèmement circonspect de certains médicaments simples... ${ }^{17}$ Pinel war im allgemeinen gegen Aderlaß und Purgieren, er war auch für eine expektative Chirurgie, er war sogar gegen den Gebrauch der Chinarinde bei Malaria! Auch dort zog er es vor, der Natur ihren Lauf zu lassen ${ }^{18}$. Dafür trat er mit um so größerer Energie für $\mathrm{Hy}$ giene, für Vorbeugung, ein und war z. B. ein begeisterter Anhänger erst der Inokulation, später der Vakzination gegen Pocken ${ }^{19}$.

12 La médecine clinique, Paris 1804, S. 441. Da dies Buch in Zusammenarbeit mit Esquirol geschrieben ist, drückt es großenteils wohl auch dessen Auffassungen aus.

13 Nosographie philosophique, $6^{\mathrm{e}}$ édition, t. I, S. LXXXVII.

14 Bichat, Anatomie générale, Paris 1830, t. I, S. XVIII.

15 Méd. clin., S. XXII.

16 Ibid., S. 15.

17 Ibid., S. 425.

18 Ibid., S. 400.

19 Ibid., S. 148. 
Pinels größter Schüler Bichat hat uns im Druck die Therapie betreffend nur sechs Seiten in den Considérations générales seiner Anatomie générale hinterlassen, wo er, nach der schon erwähnten sehr herben und durchaus skeptischen Kritik an den traditionellen Therapien, die immer der modischen allgemeinen medizinischen Theorie unterworfen worden seien, dann die Therapie ganz allgemein seiner eigenen Theorie der Sensibilitäten und Kontraktilitäten anzupassen versucht. Wir wissen aber, daß Bichat sich am Ende seines kurzen Lebens ziemlich intensiv mit Therapie beschäftigt hatte, daß ihm als echtem Arzt alles andere: Histologie, Physiologie, pathologische Anatomie (über letztere hat er ja auch nicht mehr selber publiziert) nur Vorspiel zu einer Reform der Therapie sein sollte. Glücklicherweise enthält die schöne Kollegheftsammlung des Zürcher Medizingeschichtlichen Instituts auch eine Reihe Kolleghefte eines Pariser Studenten, Louis Nicolas Jusserandot, aus dem Jahr X der Republik (1802), in deren Besitz wir durch die verständnisvolle Hilfe eines Lausanner Antiquars gelangt sind. Und unter diesen Kollegheften befindet sich auch der letzte Kurs Bichats, eben jener über Matière Médicale, der mit der 36. Lektion auf der 208. Seite jäh abbricht. Der Tod hatte dem einunddreißigjährigen Dozenten für immer den Mund geschlossen. Dieses Kollegheft erlaubt uns etwas mehr über die Entwicklung von Bichats therapeutischen Auffassungen zu berichten.

In den ersten einleitenden Vorlesungen sieht Bichat, nach Wiederholung seiner Kritik ${ }^{20}$, das Heil in einer besseren Pathologie. So wie es jetzt ist, wird dasselbe Symptom oder dasselbe Organ mit denselben Mitteln behandelt, während das Organ von verschiedenen Krankheiten befallen sein kann, die alle verschiedener Behandlungsmethoden bedürfen ${ }^{21}$. Umgekehrt können ganz verschiedene Maßnahmen dieselbe physiologische Wirkung haben.

«Les divisions de la matière médicale ont beaucoup variés. Les divisions ne peuvent qu'être encore bien insuffisantes... surtout dans les médicaments

${ }^{20}$ Diese enthält auch folgende interessante Feststellung: «Les mêmes médicaments devinrent successivement le partage des humoristes et des solidistes. Les théories changèrent, mais les médicaments restèrent les mêmes. Ils furent également employés et agirent toujours de la même manière ce qui prouve que leur action est indépendante de l'opinion des médecins, qu'on ne doit l'envisager que par une observation.»

21 «On voit que le même médicament ne peut guérir une jaunisse qui peut reconnaître différentes causes. Même chose pour la difficulté de respirer, les étouffements, les palpitations peut-êtres dues ou à une affection du cœur ou du poumon ou de la plevre.» 
qui agissent sur tout le système. C'est l'action du médicament qu'on cherche connoitre et c'est de là qu'il faut tirer les divisions médicales et non du règne animal, végétal, mineral parceque il importe peu que le médicament appartienne à un de ces règnes. Il faut savoir comment il agit et faire de là ses divisions médicales. » "C'est l'effet du médicament qu'il faut étudier. Quand on l'ignore, vaut mieux ne rien dire que d'avancer des hypothèses.»

Die Einteilungsnormen Bichats haben sich nun seit der Anatomie générale geändert. Er unterscheidet «médicaments agissants sur les fluides» und «médicaments agissants sur les solides (le plus grand nombre)». Er unterscheidet action générale und action locale, die er dann in ihren Variationen untersucht. Dann beginnt er eine Detailuntersuchung aller therapeutischen Agentien (Drogen wie Laudanum, Ipecacuanha, Helleborus; Eis: Milch usw.), die er eben nicht zu Ende führen konnte. Oft stützt er sich auf eigene Versuche am Krankenbett im Hôtel-Dieu.

Es ist schwer zu sagen, wo Bichat schließlich geendet hätte: beim Empirismus, bei der Experimentalpharmakologie MAgendies, bei einer auf ungenügenden pathologisch-physiologischen Kenntnissen aufgebauten «rationalen Therapie»? Er tendierte wohl am meisten in letzterer Richtung.

Dies war auf jeden Fall der Weg, den sein Verehrer BroussaIs, der 1816 Pinel von der Führung der Pariser Schule verdrängte, einschlug, auf dem er nicht wenig Unheil anrichtete und auf dem er schließlich scheiterte. Er reduzierte die ganze Pathologie zur Gastroentérite, die er mit Schleimsuppen und ausgiebigster lokaler Applikation von Blutegeln in der Magengegend behandelte. Was Medikamente betrifft, so kam paradoxerweise seine Haltung praktisch genau auf die seines von ihm so viel geschmähten Lehrers Pinel hinaus, wenn auch seine theoretische Begründung eine andere war (er wollte den Magen nicht noch mehr reizen). Auch er gab praktisch keine.

Skeptizismus und Empirismus treten uns in recht klarer Form in der dritten Führungsschicht der Pariser Schule, der pathologisch-anatomischen Richtung von Laennec und Louis entgegen, die ja eindeutig auf Corvisart zurückgeht. CoRvisarT empfahl zwar in seinem berühmten Buch über die Herzkrankheiten den traditionellen Aderlaß und das Purgieren, stand aber auf dem skeptischen Standpunkt: «Il est possible de prévenir quelquefois la maladie (du cœur), de la guérir, jamais» ${ }^{22}$. Dieser Standpunkt wurde wörtlich von seinem Schüler Laennec übernommen. Es ist erstaunlich, wie

${ }^{22}$ Essai sur les maladies du cœur, Paris 1806, S. LIII. 
wenig beide von der Digitalis wußten und hielten. In der Einleitung zu dem posthumen Werk seines Lehrers und Freundes Desbors de RocheFORT Cours élémentaire de matière médicale (Paris 1789) rühmt Corvisart vor allem dessen «épuration» der Materia medica.

Außerordentlich typisch ist auch die Haltung des großen CorvisartSchülers G.L.BAYLE. Sein schon erwähnter Aufsatz «Idée générale de la thérapeutique» gipfelt in der Feststellung: «La thérapeutique n'est donc point, dans tous les cas, l'art de guérir les maladies, mais bien l'art de les traiter convenablement. ${ }^{23} \mathrm{Er}$ ist also eindeutig Skeptiker. Logischerweise muß er seine Hoffnung auf die Naturheilkraft setzen. Von seinen 17 Prinzipien der Therapie unterstreichen dann auch 5 die Bedeutung derselben und der expektativen Therapie. Und trotzdem verschreibt er ziemlich viel. Die Lösung dieses Widerspruchs liegt in Prinzip XIII: "Il vaut mieux tenter un remède dont le succès est douteux que d'attendre une mort certaine» (das übrigens schon in Celsus, Buch III, Kapitel X, zu finden ist). Er ist eindeutig Empiriker. «En considérant toutes les maladies on verrait que, dans la plupart, nous ignorons l'indication curative rationelle... il en est quelquesunes qu'on traite avec succès par la méthode curative spécifique ou par la méthode empirique ce qui est presque la même chose. ${ }^{24}$ Andererseits ist auch für ihn die Pathologie die Hoffnung und Lehrmeisterin der Therapie ${ }^{25}$. Was sein Lehrer Corvisart für sein Spezialgebiet die Herzkrankheiten gesagt, gilt auch ihm für das seine, die Phthise: «La phthisie est presque toujours incurable et mortelle ${ }^{26}$.

Der große LaEnNec war ein ausgesprochener Empiriker. «Je puis assurer que cette médication a souvent une grande efficacité... Je ne sais ce que vaut la théorie sur laquelle elle repose : la chimie animale est encore trop peu avancée pour nous donner la solution d'un problème de ce genre ${ }^{27}$. Er übernahm RAsoris Behandlung der Lungenentzündung mit Brechweinstein in hohen Dosen, ohne das Geringste von dessen Theorien zu halten ${ }^{28}$. «La meilleure méthode d'apprécier une méthode de traitement, est de la juger par ses résultats» ${ }^{29}$. Er begann darum auch, Statistiken über seine

23 BAYLe, l.c., S. 610.

24 Ibid., S. 609.

25 Ibid., S. 608.

26 Ibid., S. 393.

27 Traité de l'auscultation médiate, Paris 1826, t. I, S. 182.

28 Ibid., S. 494.

29 Ibid., S. 499. 
Therapie anzulegen. Als Skeptiker zeigt er sich uns besonders in seiner Behandlung der Phthise ${ }^{30}$; er verwirft Aderlaß, Kanthariden, Blasenlegen, die erweichenden Medikamente, 26 andere Medikamente, die Gasbehandlung, und alles, was ihm bleibt, ist die «athmosphère marine artificielle». Es ist übrigens typisch, daß sich von seinen 171 Seiten über die Phthise nur 16 mit Therapie befassen. «La guérison de la phthisie tuberculeuse n'est pas au dessus des forces de la nature; mais nous devons avouer que l'art ne possède encore aucun moyen certain, d'arriver à ce but.»

Auch der einflußreiche P. Сн.A.Lours war Skeptiker. Seine Behandlung der von ihm beschriebenen «fièvre typhoide» war äußerst sparsam (Tonika, Eisbeutel, Flüssigkeitsaufnahme). Er war gegen «Derivationen» und «Revulsionen». Er war radikaler Empiriker. Die Therapie sollte nicht aus Physiologie oder Pathologie, sondern nur aus der Therapie selbst abgeleitet werden. Man warf ihm darum vor, daß er faktisch das Tierexperiment durch das am Menschen ersetzte ${ }^{31}$. Er erhob die Statistik zum grundlegenden Kriterium therapeutischer Forschung. Seine «Recherches sur les effets de la saignée» (Arch. Gén. de Méd. 1828, als Buch Paris 1835) leiteten das Ende der jahrtausendealten Aderlaßroutine ein. Wie fest verwurzelt der Aderlaß noch damals war, geht daraus hervor, daß Louis nur die geringere oder stärkere Verwendung des Aderlasses diskutieren konnte. Ihn ganz zu unterlassen, wagte niemand außer Magendie. Und an dessen Kranken wurde er dann heimlich von seinen menschenfreundlichen «internes» vorgenommen $^{32}$ ! Typisch für die Pariser Schule ist auch, daß noch 1857 der aus dem Kreis von Louis (Société médicale d'observation) stammende RenouaRD ${ }^{33}$ eine Ausrichtung der gesamten Medizin auf eine rein empirische Therapie fordern konnte.

Die Therapie der letzten Führungsschicht der Pariser Schule, der Eklektiker, ist, wie schon der Name annehmen läßt, wenig charakteristisch. Der berühmteste unter ihnen, ANDRAL, pendelte skeptisch zwischen empirischer und rationaler Therapie. Trousseau und Pidoux, die Verfasser eines vielbenützten Traité de thérapeutique, Paris 1836, sprachen sich sehr abfällig über den unsystematischen Skeptizismus vieler Kollegen (vor allem wohl Louis') aus, fanden dann aber auch in der Naturheilkraft eine Hauptstütze. Sie bekannten sich offen zum Emprismus und den spezifischen Mitteln.

30 Ibid., S. $703 \mathrm{ff}$.

31 J.L.H.Peisse, La médecine et les médecins, Paris 1857, t. I, S. 171.

32 Claude Bernard in J.M.D. Olmsted, François Magendie, New York 1944, S. 155.

33 P.V.RenouARD, Lettres philosophiques sur la médecine au XIX $X^{e}$ siècle, Paris 1857. 
Interessant ist es, den Zug zum Lokalismus bei Bretonneau ${ }^{34}$ zu beobachten. Während er im Anfang (1818) die von ihm entdeckte Diphtherie hauptsächlich allgemein (d.h. mit Quecksilberpräparaten) behandelte, ging er später mehr und mehr zur Lokalbehandlung mit Salzsäure, Silbernitrat und Tracheotomie über.

Die Pariser Skeptiker wären vielleicht auch zum Nihilismus der Wiener gelangt, hätten nicht eine Reihe wichtiger chemischer Entdeckungen und ihre Anwendung auf die Medizin neues Interesse für und Vertrauen auf die medikamentöse Behandlung erweckt, und überhaupt eine neue Ära der Therapie, beherrscht von der neu geschaffenen experimentellen Pharmakologie, eröffnet, die die alten Gegensätze zwischen «rational» und «empirisch» in gewissem Maße aufhob. Die chemischen Entdeckungen sind vor allem die der Alkaloide, die im wesentlichen dem Pariser Apotheker Jos. Pelletier und seinen Mitarbeitern zu verdanken sind. Wir müssen uns daran erinnern, daß Paris in dieser Periode nicht nur das Weltzentrum der Medizin, sondern der Naturwissenschaften überhaupt und gerade der Chemie war, die noch immer zu einem beträchtlichen Teil in den Händen der Apotheker lag. Das Morphin war schon 1804 von Sertürnner entdeckt worden. Pelletier entdeckte 1817 (mit Magendie) das Emetin, 1818 (mit Caventou) das Strychnin, 1819 (mit Caventou) Brucin und Veratrin, 1820 (mit Caventou) das Colchicin und das Chinin; P.J.Robiquet 1821 das Koffein und 1832 das Kodein ${ }^{35}$. In dieselben Jahre fällt auch die Entdeckung der Halogene ${ }^{36}$ und ihre Anwendung auf die Medizin. Zum erstenmal in der Geschichte standen dem Kliniker oder Physiologen nun eine ganze Reihe von chemisch reinen und bekannten Stoffen zur Verfügung, die er immer in der gleichen Quantität verwenden konnte, während die früheren Extrakte und Rohprodukte in ihrem Gehalt an Wirkstoffen ja dauernd außerordentlich geschwankt hatten.

Das Verdienst durch Einführung dieser Mittel eine Renaissance der Drogentherapie - damit auch zu einem gewissen Grad der Allgemeinbehandlung - herbeigeführt und eine neue Wissenschaft, die experimentelle Pharmakologie ${ }^{37}$, geschaffen zu haben, gebührt paradoxerweise dem skep-

${ }^{34}$ P.Bretonneau, De la diphthérite, Paris 1826.

35 E. Kremers and G.Urdang, History of Pharmacy, Philadelphia 1951, S. 471.

36 Ibid., S. 463.

${ }^{37}$ Als großer Vorläufer der Experimentalpharmakologie durch seine toxikologischen Arbeiten sollte allerdings J.J.WEPFEr nicht vergessen werden. Siehe HaNs Fischer, $J . J$. Wepfer, Schaff hausen 1931. 
tischsten aller Pariser Kliniker ${ }^{38}$, dem Physiologen Magendie. Man ist beinah versucht, in Hegelscher Terminologie von einem Umschlagen der Quantität (des Skeptizismus) in eine neue Qualität (die Pharmakologie) zu sprechen. Sein Formulaire pour la préparation et l'emploi de plusieurs nouveaux médicaments erschien zum erstenmal in Paris im Jahr 1821. Es erlebte eine Auflage nach der andern (wir stützen uns im folgenden hauptsächlich auf die achte aus dem Jahr 1835). Obwohl andere (wie z. B. BARBIER in der zweiten Auflage seines Traité de matière médicale, Paris 1824; Julia Fontenelle, Arch. Gén., t. IV [1824] 152) schnell denselben Weg beschritten, bleibt das Formulaire doch das grundlegende Dokument der neuen Richtung, und es sei mir darum erlaubt, es im folgenden kurz zu analysieren.

Magendie setzt im Vorwort auseinander, daß sein Werk auf der endlichen Entdeckung chemisch reiner medikamentöser Substanzen und der Überwindung des alten Vorurteils, daß die Medikamente und Gifte eine andere Wirkung beim Menschen als beim Tier haben, aufgebaut ist.

Bereits 1809 hatte Magendie experimentell den Einfluß der Brechnuß auf das Rückenmark nachgewiesen. Es ist also nicht überraschend, daß er als erstes Medikament das Strychnin behandelt. Wie bei allen Substanzen erörtert er die folgenden Punkte: Zubereitung aus Rohmaterialien, physikalische und chemische Eigenschaften, Wirkung auf das Tier, Wirkung auf den gesunden und kranken Menschen, Indikation, Anwendungsart (Rezepte für Pillen, Tinkturen, die verschiedenen Salze der Substanz). In der gleichen Art behandelt er das Brucin, Morphin, Narcotin, Narcein, Meconin und das Kodein, das er für ein besseres Schlafmittel hält als das Morphin. Es folgen Emetin (von ihm mit Pelletier 1817 gefunden), Veratrin und die Alkaloide der Chinarinde, Chinin und Cinchonin. Zusammen mit Double, Villermé und Chomel hat er die letzteren gegen beträchtliche Widerstände in die Praxis eingeführt ${ }^{39}$. Magendie hat sich nicht gescheut, 1817 die Blausäure als Hustenmittel zu empfehlen, warnte aber vor den Gefahren.

Das Jod, 1811 von Courtois entdeckt, ist seit Coindet (1819) ein vielbenutztes Kropfmittel. Bei der traditionellen Verwechslung von Kropf und Skrofulose ist es nicht verwunderlich, daß es dann auch in letzterer Krank-

38 Zu Magendies Skeptizismus OLmsted, l.c., S. 152 usw.

39 In der Dosierung war er zurückhaltender als Louis, Andral, Bally, Trousseau und MaIllot; siehe E.H. Ackerknecht, Malaria in the Upper Mississippi Valley 1760-1900, Baltimore 1945, S. 104. 
heit viel benutzt wurde ${ }^{40}$. Richon(D) hat es 1824 erfolgreich bei Syphilis gegeben. Es gilt als kräftiges menstruationsbeförderndes Mittel ${ }^{41}$. Magendie berichtet auch über seine Verwendung bei Tuberkulose, Krebs und Epilepsie. Das von BaLARD 1826 entdeckte Brom betrachtet er als Äquivalent des Jods. Das Chlor (Scheele 1774) dient vor allem als Desinfektionsmittel, auch für Wunden, und in Lungenkrankheiten.

Das Solanin, Delphinin, Gentianin und Lupulin hat er im Tierexperiment geprüft, aber keine therapeutische Verwendung für diese Substanzen gefunden. Das Krotonöl, das im Tierversuch intravenös gegeben tötet, ist ein Drastikum ${ }^{42}$. Die diuretische Wirkung des Harnstoffs wurde durch Segalas im intravenösen Tierversuch gefunden. Thridax oder Lattigsaft ist laut Dr. Françors ein Beruhigungsmittel, das auch Körpertemperatur und Pulsgeschwindigkeit vermindert.

Die Goldsalze, die man bei Syphilis und Skrofulose gegeben hat, haben sich in den intravenösen Tierversuchen des berühmten Toxikologen ORfiLA als sehr giftig erwiesen. Grenadin und Farn sind bewährte Wurmmittel. Magendie berichtet mit Zurückhaltung über die wunderbaren Resultate anderer mit Phosphor, sieht aber mit Recht voraus, daß die Entdeckung dieser Substanz im Gehirn durch Couerbe ihrer therapeutischen Verwendung eine neue Wendung geben werde.

Natrium- und Kaliumbikarbonat werden seit Darcet (1826) bei Magenbeschwerden durch Säureüberschuß gegeben. Das Digitalin des Genfer Apothekers Auguste Leroy, der auch interessante Tierversuche angestellt hat, muß erst noch geprüft werden. Das Salicin, ein von Magendie viel verwendetes Fiebermittel, ist kein Alkaloid; Magendie gibt Milchsäure bei Dyspepsie.

Das sind die neuen Mittel der Pariser Schule, die den traditionellen pflanzlichen oder mineralischen (Antimon- und Arsen- oder Quecksilber)-Präparaten zur Seite traten oder sie ersetzten. Magendies Werk wurde in

${ }^{40}$ Siehe vor allem J.G.A.Lugor, Mémoire sur l'emploi de l'iode, Paris 1829.

41 «En employant, contre une suppression de règle, l'iode chez une demoiselle dont il ne m'était permis, en aucune manière, de suspecter la bonne conduite, il est survenu un avortement au bout de trois semaines», Formulaire 1835, S. 224.

42 Die intravenöse Medikation wurde schon lange vor Magendie verwendet (siehe H. Buess, Ciba Zeitschrift, Nr. 100, März 1946). In Frankreich war die intravenöse Opiumbehandlung des Tetanus durch Percy und CH. N.Laurent schon 1797 eingeführt worden. Es besteht aber kein Zweifel, daß die intravenöse Methode durch Magendie, der sie schon sehr viel im Tierexperiment verwendet hatte, einen besonderen Auftrieb erhielt (für seine intravenöse Warmwasserbehandlung der Tollwut siehe OLMsted, l.c., S. 130). 
glänzender Weise von seinem Schüler Claude Bernard weitergeführt. Trotzdem wurde seine Methode durch Buchieim, Schmiedeberg und deren Schüler bald in größerem Umfang in Deutschland als an ihrem Entstehungsort gepflegt ${ }^{43}$. Aber das ist, wie KIPLING zu sagen pflegte, eine andere Geschichte.

43 E.H.Ackerknecht, Typen der medizinischen Ausbildung im 19. Jahrhundert, Schweiz. Med. Wschr. 87 (1957) 1361 usw. 